\section{Sexual and physical intimate partner violence among women using antenatal care in Nampula, Mozambique}

\author{
Eusébio Chaquisse, ${ }^{1-3}$ Sílvia Fraga, ${ }^{1,4}$ \\ Paula Meireles, ${ }^{1}$ Glória Macassa, ${ }^{1,5}$ \\ Joaquim Soares, ${ }^{1,6}$ Francisco Mbofana, ${ }^{3}$ \\ Henrique Barros ${ }^{1,4}$ \\ ${ }^{1}$ EPIUnit, Instituto de Saúde Pública da \\ Universidade do Porto, Portugal; \\ ${ }^{2}$ Faculdade de Ciências de Saúde, \\ University of Lúrio, Nampula, \\ Moçambique; ${ }^{3}$ National Health \\ Institute, Maputo, Mozambique; \\ ${ }^{4}$ Faculdade de Medicina, Universidade \\ do Porto, Portugal ${ }^{5}$ Department of \\ Occupational and Public Health \\ Sciences, University of Gävle, Gävle, \\ Sweden; ${ }^{6}$ Department of Health \\ Sciences, Section of Public Health \\ Sciences, Mid Sweden University, \\ Sundsvall, Sweden
}

\begin{abstract}
The aim was to estimate the prevalence of sexual and physical intimate partner violence (IPV) and its associated factors, in a sample of pregnant women using antenatal care (ANC) in Nampula province Mozambique. This cross-sectional study was carried out in six health units in Nampula, from February 2013 to January 2014. Overall, 869 participants answered the Conflict Tactics Scale 2. The lifetime and past year prevalence of sexual abuse was $49 \%$ and $46 \%$, and of physical abuse was $46 \%$ and $44 \%$, respectively. Lifetime and past year sexual abuse was significantly associated with living as a couple, alcohol drinking and having a past diagnosis of gonorrhea. Lifetime and past year physical abuse increased significantly with age and was associated with living as a couple, alcohol drinking and history with syphilis. The prevalence of lifetime and previous year violence among women using ANC was high and similar showing that most women were constantly exposed to IPV. ANC provides a window of opportunity for identifying and acting on violence against women.
\end{abstract}

\section{Introduction}

Intimate partner violence (IPV) is a major public health problem, with one in every three women worldwide ever having experienced sexual or physical IPV. ${ }^{1}$
Violence against women is a violation of human rights, it limits the social participation of women, and results in a large range of health consequences, particularly reproductive health. ${ }^{2-5}$ Women sexually or physically abused by their partners often present serious clinical conditions (e.g., mental disorders, cardiovascular diseases and hypertension) and societal adverse outcomes such as physical and psychological trauma, limited sexual reproductive control and health care seeking, illegal and unsafe abortions. ${ }^{1,6,7}$ Women who have been sexually or physically abused by their partners are twice likely to have an abortion, almost twice likely to experience depression, and, in some World Health Organization (WHO) African regions ${ }^{1}$ an increased risk for unintended pregnancy. ${ }^{8}$ Beyond the reported consequences on women health, it can be recognized that they are $16 \%$ more likely to have a low-birth-weight baby and other adverse pregnancy outcomes compared to women who have not experienced partner violence. ${ }^{1,6}$ Although studies on violence against women from Africa are scarce, available data from the WHO shows that the Africa region presents a lifetime prevalence of $36.6 \%$ of physical and/or sexual IPV among ever-partnered women. ${ }^{1,4}$ In addition, there are African countries where the prevalence against women is much higher, such as South Africa, ${ }^{9}$ and Zimbabwe, ${ }^{10}$ with estimated prevalence of physical/sexual partner violence of $55.5 \%$ and $42.8 \%$, respectively. In Mozambique, there is limited research on IPV against women, particularly in northern part of the country where the current study was carried out. However, a previous study conducted in Maputo, the capital of the country, found that $69.4 \%$ of women reported one or more types of violence during the previous year. ${ }^{11}$ In a country with high gender inequities, it is expected a high prevalence of violence against women and therefore is of paramount important to implement strategies to monitor the problem in these contexts, to intend to reduce sexual and physical consequences of violence against women.

In developed countries there is evidence that screening IPV during pregnancy might be of great contribution to plan interventions in reproductive health services and therefore to prevent and minimize the impact of such violence. ${ }^{10,12}$ Antenatal care can provide an important point of contact where women can be screened for physical and sexual violence and, for those who report IPV, referred to services that can assist them. This could serve as a supplemental screening tool to detect women who have been victims of IPV, these could allow to formulate public health intervention for
Correspondence: Henrique Barros, Alameda Professor Hernâni Monteiro, 4200-319 Porto, Portugal. Tel.: +351.225513652 -

Fax: +351.225513653

E-mail: hbarros@med.up.pt

Key words: Intimate partner violence; prevalence; pregnant women; reproductive health; antenatal care; Nampula, Mozambique.

Funding: the University of Lúrio (Universidade de Lúrio) received funding for this study from the Camões - Instituto da Cooperação e da Lingua, I. P and from Fundo Nacional de Investigação (projecto $\mathrm{nr}$ 134Inv/FNI).

Contributions: EC and SF drafted the manuscript and performed the data analysis. PM, GM and JS reviewed the manuscript for important intellectual content. FM and HB participated in the study design and reviewed the manuscript for important intellectual content.

Conflict of interest: the authors declare no conflict of interest.

Received for publication: 17 July 2017.

Revision received: 22 August 2017.

Accepted for publication: 22 August 2017.

This work is licensed under a Creative Commons Attribution NonCommercial 4.0 License (CC BY-NC 4.0).

(C) Copyright E. Chaquisse et al., 2018

Licensee PAGEPress, Italy

Journal of Public Health in Africa 2018; 9:744

doi:10.4081/jphia.2018.744

detection and prevention of violence. ${ }^{8,13}$ In the African context, asking women about violence during antenatal care may represent a unique opportunity to reach these women and to assess the magnitude of this issue though in such context we can expect women to delay or omit antenatal care.

Thus, this study aimed to estimate the prevalence of sexual and physical intimate partner violence against women and its associated factors, in a sample of women using antenatal care in Nampula province, in Mozambique.

\section{Materials and Methods}

\section{Participants and setting}

The participants were recruited in six health units in Nampula Province, Mozambique (Hospital Geral de Marrere, Centro de Saúde 25 de Setembro, Centro de Saúde $1^{\circ}$ de Maio, Centro de Saúde de Muhala Expansão, Centro de Saúde de Namicopo and Centro de Saúde Hospital 
Psiquiátrico), from February 2013 to January 2014. Pregnant women, who visited these primary health care facilities for their first prenatal appointment, were eligible to participate in the study.

\section{Design/procedure}

In each health facility, maternal and child health nurses were trained to perform the study procedures, namely to conduct the interviews using a structured questionnaire. After the training, a pilot study was conducted to allow nurses to be familiarized with the questionnaire as well as to harmonize procedures. The sampling procedure consisted in inviting to participate one in every three women attending their first prenatal appointment. 1216 women gave their informed consent among them 946 were or have been in a relationship lasting more than one month and were, therefore, eligible to answer the Conflict Tactics Scale 2 (CTS2).

Of the 946 women, 77 were excluded from the analysis due to missing information on violence measures. Therefore, 869 women were included in the final analysis, among those, 40 women were not at the moment of the interview in a relationship.

\section{Measures}

The questionnaire was administered by trained nurses and information was collected on sociodemographic characteristics, tobacco, alcohol and illegal drugs use, sexual behavior (age at the first intercourse, number of sexual partners in the last 6 months), gestational age at the first appointment, history of neonatal deaths, and also diagnosis of sexually transmitted infections.

IPV was assessed using the conflict tactics scales. ${ }^{14}$ Women were asked whether they had been victims of various types of IPV. The CTS2 covers psychological aggression, physical assault, sexual coercion, physical assault with injury. The acts may have occurred once, twice, 3 to 5,6 to 10,11 to 20 or more than 20 times during the previous year, had not occurred during the previous year but before or never occurred.

\section{Data analyses}

For analysis, maternal age was categorized in five categories ( $>18,18-20,21-25$, $26-29$, $\geq 30$ years), education was categorized according to the Mozambique education system (no education/did not finish primary school, primary school including the first and second degree, secondary school, and pre-university and university degree), marital status was categorized in married or in cohabitation vs. single or separated, and occupation was classified in three cate- gories: housewife or unemployed, employed or farmer, and student

Information was obtained on the age at the first sexual intercourse and then categorised as $\leq 14,15-19,>19$ years, having more than one sexual partner in the previous 6 months was recoded in yes or no. Parity was classified as $0,1,2,3,4$ or more, the gestational age at the first appointment was recoded in $\leq 14$ weeks, $15-27$ weeks, $\geq 28$ weeks, roughly corresponding to trimesters. For history of neonatal deaths women were categorized as primigravidae, multigravidae with no neonatal deaths and multigravidae with neonatal deaths. Women were also asked about previous diagnosis of HIV, syphilis and gonorrhoea.

In this analysis we only used physical assault acts (e.g., beat up), sexual coercion acts (e.g., threaten to have sex) and injury (e.g., bruises). For the present analysis we considered that women had been abused during the previous year, if they disclosed at least one occurrence of abuse during that period, independently of chronicity.

\section{Statistical analysis}

The data entrance was double checked. The Chi-square test was used to compare proportions. Significance level was set at 0.05 . The odds ratio (OR) and respective $95 \%$ confidence intervals $(95 \% \mathrm{CI})$ were calculated through logistic regression, with adjustment for the potential confounders. The data analysis was performed using the statistical software SPSS, version 22.

\section{Ethical considerations}

Participants were informed of study procedures, benefits and risks of participation, and provided written informed consent for interviews. All study procedures were taken to ensure confidentiality and did not change the health care provision routines. The interviews were conducted in a consultation office, where pregnant women followed all the clinical recommended procedures. Health care and support services were offered for victims of either sexual or physical violence. ${ }^{15}$ The study was approved by Ministry of Health of Mozambique and by National Bioethics Committee of Mozambique.

\section{Results}

In this sample of 869 pregnant women the lifetime prevalence of IPV sexual abuse was $48.8 \%$ and that of physical abuse was $46.0 \%$. During the previous year a similar prevalence was found both for sexual abuse (45.6\%) and physical abuse (43.4\%). The sample characteristics are presented in
Table 1. Briefly, almost two thirds of women were 25 years old or younger, 599 $(68.9 \%)$ had primary school or less, 678 $(78.0 \%)$ were housewives or unemployed and $791(91 \%)$ lived as a couple. As for their sexual life, approximately half were 15 to 19 years old in their first sexual intercourse and $735(84.6 \%)$ had only one sexual partner in the previous six months. In terms of reproductive characteristics, $270(31.1 \%)$ were nulliparous and almost $20 \%(n=173)$ had had four or more deliveries, 61 (7.0\%) had a neonatal death before. As for gestational age at the time of the first prenatal appointment, $63(7.2 \%)$ women were 14 or less weeks, $455(52.4 \%)$ were between 15 to 27 weeks and 334 (38.4\%) were 28 or more weeks. Drinking alcohol was reported by $73(8.4 \%)$ women. Previous diagnosis of HIV was reported by 60 (6.9\%) women, of syphilis by $35(4.0 \%)$ and of gonorrhoea by $21(2.4 \%)$ women. Sexual and physical IPV occurred in every age group. However, physical violence tended to increase significantly with age after the age of 21-25 years, and this remained after adjusting for education and living in couple (Table 2). More educated women (secondary school and pre-university and university degree) were less likely to have experienced sexual and physical IPV, both during lifetime and in the previous year (Table 2). As well, students were significantly less likely to report both lifetime and past year sexual IPV, this association was not statistically significant for physical IPV. Women living as a couple had approximately twice the odds of reporting sexual IPV both lifetime and past year. This association was not statistically significant for physical IPV. History of alcohol consumption was significantly associated with reporting sexual and physical IPV at any time period. There were no significant differences in the prevalence of sexual abuse and physical violence among women who had one partner compared with those who had more than one partner during the previous six months. Women with their first sexual intercourse after the age of 15 years were significantly less likely to be physically abused both in lifetime and in the previous year, as well as those with their first sexual intercourse after the age of 19 were less likely to report lifetime physical abuse. There was a crude association between multiparity with no deaths and with one or more neonatal deaths and physical abuse, both in lifetime and in the previous year (Table 1), but this association lost significance after adjustments (Table 2). No associations were found for any other reproductive characteristics.

Pregnant women who reported a previous diagnosis of syphilis had twice the odds 
Table 1. Prevalence of sexual abuse, physical abuse and injury according to sociodemographics and behavioural characteristics, adverse maternal outcomes and sexually transmitted infections; 2013/2014, Nampula, Mozambique.

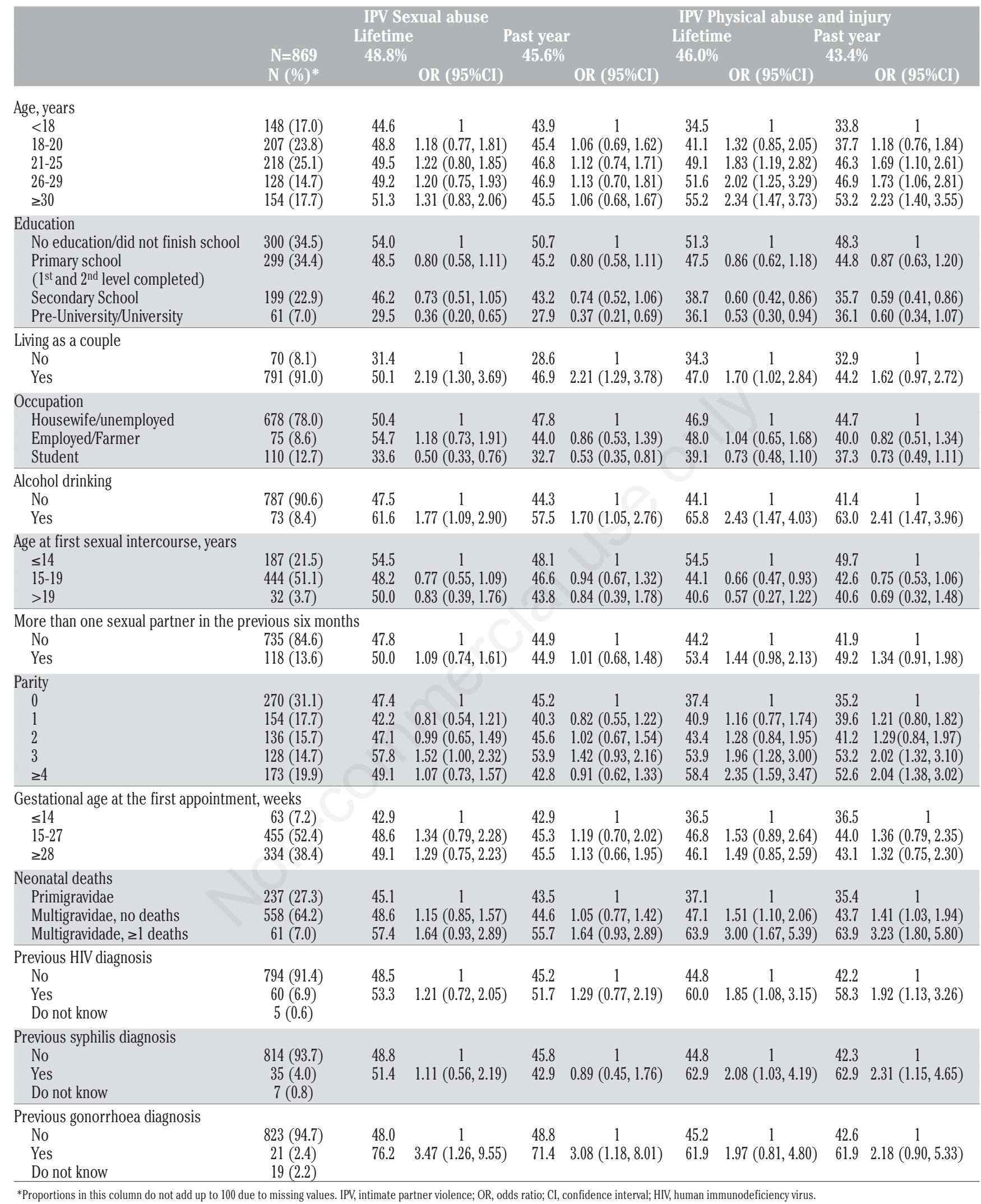

Proportions in this column do not add up to 100 due to missing values. IPV, intimate partner violence; OR, odds ratio; $\mathrm{Cl}$, confidence interval; HIV, human immunodeficiency virus. 
of reporting physical violence in their lifetime $(\mathrm{aOR}=2.07 ; 95 \%$ IC: $0.99-4.34)$, and in the previous year $(\mathrm{aOR}=2.30 ; 95 \%$ IC: 1.10-4.81). Similar findings were found for previous gonorrhoea diagnosis and sexual abuse in lifetime and in the previous year. Although there was a crude significant association with previous HIV diagnosis and sexual abuse, it lost significance after adjusting for age, education and living in couple.

Table 2. Association (Adjusted OR (95\% CI) between sexual abuse, physical abuse and injury, sociodemographics and behavioural characteristics, adverse maternal outcomes and sexually transmitted infections; 2013/2014, Nampula, Mozambique.

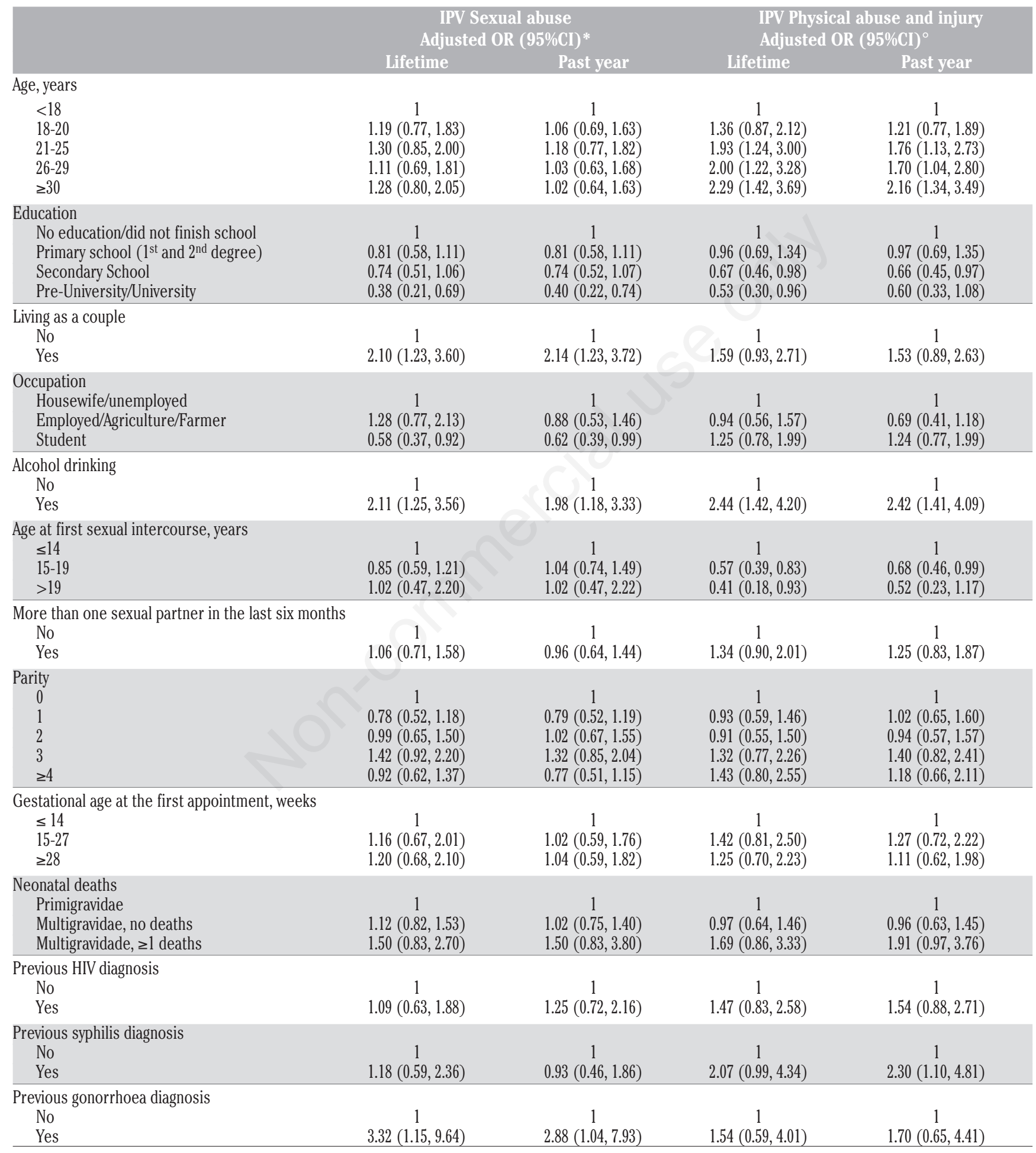

*Adjusted for education and living in couple; ${ }^{\circ}$ Adjusted for age, education and living in couple. IPV, intimate partner violence; $\mathrm{OR}$, odds ratio; $\mathrm{CI}$, confidence interval; HIV, human immunodeficiency virus. 


\section{Discussion}

The lifetime prevalence of sexual and physical IPV was high, with $48.8 \%$ of pregnant women experiencing sexual violence and $46.0 \%$ physical abuse during their lifetime. A similar prevalence was found both for sexual abuse $(45.6 \%)$ and physical abuse (43.4\%) during the previous year, indicating that abuse is a continuum in life. These high prevalence are consistent with studies conducted worldwide, ${ }^{16}$ and in Africa showing an overall prevalence of 20\%-70\%. ${ }^{1,4,17}$ Similar results were found in the neighbouring Zimbabwe where the prevalence was estimated at $46.2 \%$ for physical and/or sexual violence. ${ }^{10}$ The high prevalence observed in this study of IPV could be related to the patriarchal ideology in traditional societies such as found in Mozambique, where gender roles are distorted to justify violence against women, who are mostly assigned an inferior role to men. ${ }^{18,19}$ In addition, the low level of education, young age and low socio-economic status and HIV diagnosis could contribute for higher prevalence of IPV. ${ }^{4}$ On the other hand, more educated women were shown to report less violence, supporting that increasing women's education empowers them.

Physical abuse increased with age, being approximately twice as likely in women aged 21 years and over, even after adjusting for education and living with a partner. These results are similar to those of a study in Rwanda, which shows that women aged $26-34$ were more likely to suffer physical abuse, ${ }^{20}$ suggesting that males may use violence between partners to control sexual decision making and coercive sex. $^{21}$

Initiating sexual intercourse at age 15 and older seems to be a protective factor, with an inverse relationship between age at the onset of sexual intercourse and the possibility of physical violence, and this trend was maintained when adjusted for education and living with the partner. This finding is consistent with other studies which show the importance of a delay in the onset of sexual intercourse as a protective factor, ${ }^{22-24}$ on other hand the young age has been considered as a most factor associated with increased likelihood of IPV. ${ }^{25}$

A significant association was found between exposure to sexual violence and diagnosis of gonorrhoea and also between physical violence and syphilis diagnosis. It is known that IPV is concurrent with a higher risk of sexually transmitted diseases (STD), as well as a reduction in women's use of contraception, ${ }^{26,27}$ due to violent behaviour by male partner. ${ }^{28}$ However, this result must be read carefully as a small number of women reported a previous diagnosis of these STD.

Multigravidae with one or more stillbirths were at a 3-fold increased risk for physical abuse compared to primigravidae, although after adjustment the strength of the association decreased. These findings seem to show that exposure to physical violence has an impact in pregnancy outcomes. In this study we were not able to determine if reported violence has occurred during pregnancy, however, there is strong evidence from African studies that a history of experiencing abuse in lifetime and in the previous 12 months is significantly associated with IPV in pregnancy or just before pregnancy. $4,29,30$

\section{Strengths and limitations}

The Conflict Tactics Scales has been used frequently in many studies since 1972 . The CTS is a measurement tool for research program of the family conflicts, it was revised by Murray Straus et al, in 1979. The theoretical basis of the CTS is conflict theory, assuming that conflict is an inevitable part of all human. The CTS2 replaces the matrix format developed for research with military families, ${ }^{14}$ and the instrument has been adapted to be applied on researches in Mozambique context. ${ }^{11}$

Although the questionnaires were administered to pregnant women using antenatal care, violence during pregnancy was not assessed. However, a high proportion of women reported sexual and physical abuse during the previous year, and therefore, we may speculate that pregnancy could not stop the violence episodes. Also, we sampled a specific group of women that seek for the antenatal care. It is known that in such contexts, women tend to avoid antenatal care and sometimes deliver at home. Due to its cross-sectional nature, the study cannot provide causal links and there is possible information/disclosure bias. Thus, we might expect a high prevalence of violence in the general population and also an underestimation of the associations found.

\section{Conclusions}

This study found a high prevalence of lifetime and past year violence among women using antenatal care in Mozambique. Antenatal care provides a window of opportunity for identifying and acting on violence against women. Therefore, it is necessary to define specific strategies to support women in the context of Mozambique.

\section{References}

1. World Health Organization. Global and regional estimates of violence against women: prevalence and health effects of intimate partner violence and nonpartner sexual violence. In: WHO, ed. Geneva 27, Switzerland: WHO documents prodution services; 2014. Available from: http://apps.who.int/iris/ bitstream/10665/85239/1/97892415646 25 eng.pdf.

2. Silva EP, Ludermir AB, Araujo TV, Valongueiro SA. Frequency and pattern of intimate partner violence before, during and after pregnancy. Rev Saude Publica. 2011;45:1044-53.

3. Van Parys AS, Deschepper E, Michielsen K, et al. Prevalence and evolution of intimate partner violence before and during pregnancy: a crosssectional study. BMC Pregnancy Childbirth 2014;14:294.

4. Shamu S, Abrahams N, Temmerman M, et al. A systematic review of African studies on intimate partner violence against pregnant women: prevalence and risk factors. PLoS One 2011;6: e17591.

5. Campbell JC. Health consequences of intimate partner violence. Lancet 2002;359:1331-6.

6. Heise L, Ellsberg M, Gottmoeller M. A global overview of gender-based violence. Int J Gynaecol Obstet 2002; 78:S5-14.

7. Lown EA, Vega WA. Intimate partner violence and health: self-assessed health, chronic health, and somatic symptoms among Mexican American women. Psychosom Med 2001;63:35260.

8. Goodwin MM, Gazmararian JA, Johnson CH, et al. Pregnancy intendedness and physical abuse around the time of pregnancy: findings from the pregnancy risk assessment monitoring system, 1996-1997. PRAMS Working Group. Pregnancy Risk Assessment Monitoring System. Matern Child Health J 2000;4:85-92.

9. Dunkle KL, Jewkes RK, Brown HC, et al. Prevalence and patterns of genderbased violence and revictimization among women attending antenatal clinics in Soweto, South Africa. Am J Epidemiol 2004;160:230-9.

10. Shamu S, Abrahams N, Zarowsky C, et al. Intimate partner violence during pregnancy in Zimbabwe: a cross-sectional study of prevalence, predictors 
and associations with HIV. Trop Med Int Health 2013;18:696-711.

11. Zacarias AE, Macassa G, Svanstrom L, et al. Intimate partner violence against women in Maputo city, Mozambique. BMC Int Health Hum Rights 2012;12:35.

12. Miller E, McCauley HL, Tancredi DJ, et al. Recent reproductive coercion and unintended pregnancy among female family planning clients. Contraception 2014;89:122-8.

13. Alio AP, Clayton HB, Garba M, et al. Spousal concordance in attitudes toward violence and reported physical abuse in African couples. J Interpers Violence 2011;26:2790-810.

14. Straus MA, Hamby SL, MacCoy SB, Sugarman DB. Revised conflicts tactics scales (CTS2). J Fam Issues 1996;17: 283-316.

15. World Health Organization. Preventing violence: A guide to implementing the recommendations of the world report on violence and health. In: World Health Organization, ed. Geneva, Switzerlend: WHO; 2014. Available from: http://whqlibdoc.who.int/publications/2 004/9241592079.pdf?ua=1

16. Sarkar NN. The impact of intimate partner violence on women's reproductive health and pregnancy outcome. J Obstet Gynaecol 2008;28:266-71.

17. Ononokpono DN, Azfredrick EC. Intimate partner violence and the uti- lization of maternal health care services in Nigeria. Health Care Women Int. 2014;35:973-89.

18. Antai D. Traumatic physical health consequences of intimate partner violence against women: what is the role of community-level factors? BMC Womens Health 2011;11:56.

19. Antai D, Adaji S. Community-level influences on women's experience of intimate partner violence and terminated pregnancy in Nigeria: a multilevel analysis. BMC Pregnancy Childbirth 2012; $12: 128$

20. Ntaganira J, Muula AS, Masaisa F, et al. Intimate partner violence among pregnant women in Rwanda. BMC Womens Health 2008;8:17.

21. van der Straten A, King R, Grinstead O, et al. Couple communication, sexual coercion and HIV risk reduction in Kigali, Rwanda. Aids 1995;9:935-44.

22. Rickert VI, Wiemann CM, Harrykissoon SD, et al. The relationship among demographics, reproductive characteristics, and intimate partner violence. Am J Obstet Gynecol 2002; 187:1002-7.

23. Sareen J, Pagura J, Grant B. Is intimate partner violence associated with HIV infection among women in the United States? Gen Hosp Psychiatry 2009;31: 274-8.

24. Li Q, Kirby RS, Sigler RT, et al. A multilevel analysis of individual, house- hold, and neighborhood correlates of intimate partner violence among lowincome pregnant women in Jefferson county, Alabama. Am J Public Health 2010;100:531-9.

25. Organization WH. Understanding and addressing violence against women: Intimate partner violence; 2012.

26. Maxwell L, Devries K, Zionts D, et al. Estimating the effect of intimate partner violence on women's use of contraception: a systematic review and metaanalysis. PLoS One 2015;10:e0118234.

27. Decker MR, Miller E, McCauley HL, et al. Recent partner violence and sexual and drug-related STI/HIV risk among adolescent and young adult women attending family planning clinics. Sex Transm Infect 2014;90:145-9.

28. Rickert VI, Wiemann CM, Vaughan RD, White JW. Rates and risk factors for sexual violence among an ethnically diverse sample of adolescents. Arch Pediatr Adolesc Med 2004;158:1132-9.

29. Cripe SM, Sanchez SE, Perales MT, et al. Association of intimate partner physical and sexual violence with unintended pregnancy among pregnant women in Peru. Int J Gynaecol Obstet 2008; 100:104-8.

30. Hess KL, Javanbakht M, Brown JM, et al. Intimate partner violence and sexually transmitted infections among young adult women. Sex Transm Dis 2012;39: 366-71. 